\title{
UNTERNEHMENSVERANTWORTLICHKEIT IM KARTELLRECHT
}

\author{
Corporate responsibility in antitrust law
}

\author{
NADJA MÜLLER*
}

\begin{abstract}
The functional concept of a corporate responsibilty in European antitrust law, which is independent of a company's legal form, is increasingly gaining acceptance not only at the European level but also at the national level; it allows corporate sanctioning in line with the economic and socio-political role of companies in today's world.
\end{abstract}

Keywords: corporate responsibilty, antitrust law, economic unit, sanctioning model, role model

\section{EINLEITUNG}

Dem europäischen Kartellsanktionenrecht liegt ein eigenständiger funktionaler Unternehmensbegriff zugrunde, der eine effektive Durchsetzung des Wettbewerbsrechts sicherstellen soll und Ausgangspunkt einer Entwicklung darstellt, die sich nach dem Willen des europäischen Normgebers auch in den nationalen Rechtsordnungen niederschlagen soll. Ein Anfang wurde dabei im deutschen Recht im Rahmen der 9. GWB-Novelle gemacht, wobei das deutsche Kartellsanktionenrecht einen eigenen Weg der Sanktionierung geht und den europäischen Unternehmensbegriff nicht vollständig übernimmt. Ob dies der Schlusspunkt der Entwicklung sein wird, bleibt abzuwarten, ist aber zu bezweifeln.

\section{AUSGANGSPUNKT: FUNKTIONALER UNTERNEHMENSBEGRIFF}

Ausgangspunkt der Bußgeldverantwortung im europäischen Kartellrecht ist nach der Konzeption der Art. 101 und 102 AEUV iVm Art. 23 VO 1/2003 das Unternehmen bzw. die Unternehmensvereinigung. Nachdem die europäische Kommission und die Gerichte zunächst - angelehnt an den Begriff der juristischen Person im nationalen Recht - von einem materiell-institutionellen Unternehmensbegriff ausgingen ${ }^{1}$, entwickelte der EuGH in Abgrenzung dazu einen unionsrechtlich autonomen Unternehmensbegriff ${ }^{2}$, dem eine wirtschaftliche statt rechtliche und eine mehr funktionale

* Rechtsanwältin und Habilitandin am Lehrstuhl Prof. Dr. Dr. h.c. Gerhard Dannecker, Universität Heidelberg.

1 EuGH, 13. 07. 1962, verb. Rs. 17/61 und 20/61, Slg. 1962, 659, 687 (Klöckner Werke und Hoesch AG); so auch 13. 07. 1962, Rs. 19/61, Slg. 1962, 675, 750 (Mannesmann).

2 Schlussanträge GA BOT vom 26. 20. 2010, Rs. C-201/09 P u. C-216/09 P, Rn. 175 (Arcelor Mittal). 
statt formale Unternehmenskonzeption zugrunde liegt. Danach gilt, dass „,im Rahmen des Wettbewerbsrechts [...] der Begriff des Unternehmens jede eine wirtschaftliche Tätigkeit ausübende Einheit, unabhängig von ihrer Rechtsform und der Art ihrer Finanzierung “, umfasst. ${ }^{3}$

\section{DAS KONZEPT DER WIRTSCHAFTLICHEN EINHEIT}

Kommission und Gerichte betrachten also die selbstständige Ausübung einer wirtschaftlichen Tätigkeit als maßgebliche Voraussetzung für die Bejahung der Unternehmenseigenschaft. Daher kommt es entscheidend auf die tatsächliche Möglichkeit der Beeinflussung der Wettbewerbsbedingungen am Markt an. Der Unternehmensbegriff wird außerdem rechtsformunabhängig gebildet, was bedeutet, dass ein Unternehmen - sprich: die wirtschaftlich tätige Einheit - rechtlich aus mehreren natürlichen oder juristischen Personen bestehen kann. ${ }^{4}$ Verstößt eine solche wirtschaftliche Einheit gegen die Wettbewerbsregeln, hat sie nach dem Grundsatz der persönlichen Verantwortlichkeit für diese Zuwiderhandlung einzustehen. ${ }^{5}$ Generalanwältin Kokott hat im Jahr 2007 in Sachen ETI den Grundsatz der persönlichen Verantwortlichkeit wie folgt erläutert: „Aus dem Sanktionscharakter der von Wettbewerbsbehörden zur Ahndung von Kartellvergehen verhängten Maßnahmen - insbesondere Geldbußen - folgt, dass es sich um einen dem Strafrecht zumindest verwandten Bereich handelt. Maßgeblich für die Zurechnung von Kartellvergehen ist deshalb der Grundsatz der persönlichen Verantwortlichkeit, der seinerseits im Rechtsstaatsprinzip und im Schuldprinzip verankert ist. Persönliche Verantwortlichkeit bedeutet, dass ein Kartellvergehen grundsätzlich derjenigen natürlichen oder juristischen Person zuzurechnen ist, die das an dem Kartell beteiligte Unternehmen betreibt; mit anderen Worten haftet der Rechtsträger dieses Unternehmens. Durch die Orientierung an der persönlichen Verantwortlichkeit wird in der Regel zugleich ein Beitrag zur effektiven Durchsetzung der Wettbewerbsregeln geleistet, hat doch der Betreiber eines Unternehmens auch den maßgeblichen Einfluss auf dessen Marktverhalten; dieses Verhalten soll er unter dem Eindruck der verhängten Sanktionen neu ausrichten, damit das Unternehmen sich künftig wettbewerbskonform verhält. Gleichzeitig hat die Sanktion eine generalpräventive Wirkung, indem sie auch andere Wirtschaftsteilnehmer von der Begehung von Kartellvergehen abschreckt. " ${ }^{\circ 6}$

3 EuGH, 23.04.1991, Rs. C-41/90, Slg. 1991, I-1979 Rn. 21 (Höfner und Elser); DANNECKER Christoph - DANNECKER Gerhard: Europäische und verfassungsrechtliche Vorgaben für das materielle und formelle Unternehmensstrafrecht. NZWiSt, 2016, 162, 167.

4 EuGH, 20. 01. 2011, Rs. C-90/09 P, www.curia.eu, Rn. 35 (General Quimica u. a.).

5 Siehe nur EuGH, 10. 04. 2014, Rs. C-231/11 bis C-233/11 P, www.curia.eu, Rn. $42-44$ (Siemens Österreich); 29. 03. 2011, Rs. C.201/09 P u. C-216/09 P, www.curia.eu, Rn. 95 (ArcelorMittal Luxembourg).

6 Schlussanträge GA Kokott vom 03. 07. 2007, Rs. C-280/06, Slg. 2007, I-10893 Rn. 71f. (ETI); Hervorhebungen durch die Verfasserin; s.a. EuGH, 27. 04. 2017, Rs. C-516/15 P, www.curia.eu, Rn. 52 (Akzo Nobel); 29. 03. 2011, Rs. C-201/09 P und C-216/09 P, www.curia.eu, Rn. 95 (ArcelorMittal Luxembourg). 
Das Abstellen auf die wirtschaftliche Einheit führt dabei einerseits dazu, dass wettbewerbswidrige Absprachen bzw. Fusionen und Umstrukturierungen innerhalb dieser Einheit aus dem Wettbewerbsrecht auszunehmen sind, da sie nicht „,nach außen" wirken und somit keine nachteilige Beeinflussung des Marktgeschehens bewirken (sog. Konzernprivileg). ${ }^{7}$

Andererseits kommt der wirtschaftliche Unternehmensbegriff insbesondere bei der Ahndung von Kartellverstößen eines Konzerns zum Tragen, wenn also die wirtschaftliche Einheit aus mehreren Rechtsträgern besteht. In solchen Fällen geht der EuGH in ständiger Rechtsprechung davon aus, dass einer Muttergesellschaft das Verhalten ihrer Tochtergesellschaft zugerechnet werden kann, und zwar insbesondere dann, wenn die Tochtergesellschaft trotz eigener Rechtspersönlichkeit ihr Marktverhalten nicht selbstständig bestimmt, sondern im Wesentlichen Weisungen der Muttergesellschaft befolgt. Dies wird dann angenommen, wenn wirtschaftliche, organisatorische und rechtliche Beziehungen zwischen den beiden Rechtssubjekten bestehen und darüber eine Einflussnahme der Mutter- auf die Tochtergesellschaft stattfindet (sog. Konzept des bestimmenden Einflusses). ${ }^{8}$

Hält die Muttergesellschaft (nahezu) das gesamte Kapital der kartellrechtswidrig handelnden Tochtergesellschaft, besteht laut Kommission und den Gerichten eine widerlegliche Vermutung dafür, dass die Muttergesellschaft tatsächlich einen bestimmenden Einfluss auf ihre Tochtergesellschaft ausübt. In einem solchen Fall kann die Kommission demnach die Muttergesellschaft für das Verhalten der Tochtergesellschaft zur Verantwortung ziehen, ohne zusätzliche Beweise für eine tatsächliche Einflussnahme vorbringen zu müssen. ${ }^{9}$

Bei fehlender Autonomie und Weisungsabhängigkeit kann eine wirtschaftliche Einheit auch zwischen gleichgeordneten Konzerngesellschaften bzw. Schwestergesellschaften angenommen werden. ${ }^{10}$ Eine wirtschaftliche Einheit kann darüber hinaus zwischen Mutter-, Tochter- und Enkelgesellschaft angenommen werden, wenn

7 Kersting, Christian: Die Rechtsprechung des EuGH zur Bußgeldhaftung in der wirtschaftlichen Einheit. $W u W, 2014,1156,1158$.

8 DANNECKER, Gerhard - MÜLLER, Nadja: Kartellstraf- und -ordnungswidrigkeitenrecht. In: WABNITZ, Heinz-Bernd - JANOvSKY, Thomas - SCHMITT, Lothar (Hrsg.): Handbuch Wirtschafts- und Steuerstrafrecht. 5. Aufl., 2020, Kap. 19 Rn. 230 f.; EuGH, 28. 06. 2005, Rs. C-189/02 P u.a., www.curia.eu, Rn. 119 f. (Dansk Rørindustri); EuG, 12. 07. 2018, Rs. T-419/14, EuZW, 2018, 858, 869 Rn. 143 (Goldman Sachs Inc.); EuGH, 27. 04. 2017 , Rs. C-516/15 P, www.curia.eu, Rn. 52 (Akzo Nobel).

9 DANNECKER - MÜller (2020): a.a.O., Kap. 19 Rn. 230; EuGH, 8.5.2013, Rs. C-508/11 P, www.curia.eu, Rn. 67 (ENI SpA); EuG, 13. 7. 2011, Rs. T-138/07, www.curia.eu, Rn. 73 (Schindler Holding); EuGH, 14. 07. 1972, Rs. 48/69, www.curia.eu, Rn. 131 bis 133 (Imperial Chemical Industries); 25. 10. 1983, Rs. C-107/82, www.curia.eu, Rn. 49 bis 53 (AEG-Telefunken); 17. 09. 2015, Rs. C-597/13 P, www.curia.eu, Rn. 35 (Total).

10 EuGH, 02. 10. 2003, Rs. C-196/99, Slg. 2003, I-11005 Rn. 96 ff. (Aristain); EuG, 27.11. 2014, Rs. T-517/09, www.curia.eu, Rn. 59 (Alstom/Kommission). 
eine Muttergesellschaft beispielsweise (nahezu) 100\% des Kapitals der Tochtergesellschaft hält, die wiederum Alleingesellschafterin der Enkelgesellschaft ist. ${ }^{11}$

Um zu verhindern, dass sich Unternehmen durch Umwandlungen, Umstrukturierungen oder Liquidation der Bebußung entziehen, wird auf die wirtschaftliche Einheit auch in Fällen der Rechtsnachfolge zurückgegriffen. ${ }^{12}$ Ein solcher Rückgriff im Rahmen der Rechtsnachfolge ist nach Auffassung des EuGH in Sachen Modelo Continente Hipermercados selbst dann möglich, wenn noch kein Bußgeldverfahren eröffnet bzw. noch keine Geldbuße festgesetzt oder gar rechtskräftig geworden ist. Im fraglichen Fall ging der Gerichtshof davon aus, dass die bußgeldrechtliche Zahlungspflicht schon als ungewisse Verbindlichkeit auf den Rechtsnachfolger übergeht. ${ }^{13}$

\section{BUßGELDRECHTLICHE VERANTWORTUNG UND HAFTUNG IN DER WIRT- SCHAFTLICHEN EINHEIT}

Das Konzept der wirtschaftlichen Einheit vermeidet damit im Ergebnis infolge seines rechtsformunabhängigen Charakters Zurechnungsprobleme zwischen den Rechtsträgern im Konzern: Wird ein Kartellrechtsverstoß aus der wirtschaftlichen Einheit heraus begangen, ist sie dafür persönlich verantwortlich. Allerdings stößt das Konzept naturgemäß dort an seine Grenzen, wo aus rechtlichen Gründen nur auf eine juristische Person zugegriffen werden kann, so bei der Zustellung (die wirtschaftliche Einheit hat keinen „Briefkasten“) oder Vollstreckung eines Bußgeldbescheids. ${ }^{14}$

Das bedeutet, dass die Kommission im Rahmen des Kartellordnungswidrigkeitenverfahrens zwar zunächst die aus mehreren Rechtsträgern bestehende wirtschaftliche Einheit als Verantwortliche für den Kartellverstoß ahnden kann, aber sich spätestens, wenn es um die praktische Versendung der Bußgeldbescheide geht, darauf festlegen muss, welchen Rechtsträgern aus der wirtschaftlichen Einheit sie diese Bescheide zustellen will, bzw. wer das festgesetzte Bußgeld letztlich bezahlen muss. ${ }^{15}$

In der Praxis geht die Kommission hier zwei verschiedene Wege: Zunächst kann sie, unter Berücksichtigung des Schuldgrundsatzes, nach dem Grundsatz der persönlichen Verantwortlichkeit „maßgeschneiderte Bußgelder“ gegen alle, gegen mehrere

11 DANNECKER - MÜLler (2020): a.a.O., Kap. 19 Rn. 234; Kersting, Christian: Wettbewerbsrechtliche Haftung im Konzern. Der Konzern, 2011, 445 f.; EuGH, 08. 05. 2013, Rs. C-90/09 P, Slg. 2011, I-1, Rn. 86 ff. (General Quimica); Siehe KOM, 11. 06. 2008, COMP/38.695 Rz. 396 (Natriumchlorat), wo eine Kapitalbeteiligung von 97\% als ausreichend betrachtet wurde.

12 Siehe nur Schlussanträge GA KOKOTT, v. 03. 07. 2007, Rs. C 280/06, Slg. 2007, I 10893, Rn. 71 ff. (ETI u. a.).

13 DANNECKER - MÜller (2020): a.a.O., Kap. 19 Rn. 237; EuGH, 05.03.2015, Rs. C343/13, www.curia.eu, Rz. 24 ff. (Modelo Continente Hipermercados).

14 EuGH, 27. 04. 2017, Rs. C-516/15 P, www.curia.eu, Rn. 50 (Akzo Nobel); DANNECKER DANNECKER: NZWiSt, 2016, 162, 168.

15 Siehe nur KOMM, 08. 07. 2009, COMP/39.401, ABl. 2009 C 248 Rn. 270 ff. (E.ON/GDF); EuGH, 27. 04. 2017, Rs. C-516/15 P, www.curia.eu, Rn. 48 ff. (Akzo Nobel). 
oder gegen nur einen beteiligten Rechtsträger verhängen (strafrechtliche Verantwortung im weiteren Sinne). ${ }^{16}$ Welchen oder wie viele Rechtsträger aus der wirtschaftlichen Einheit die Kommission dabei als Bußgeldschuldner auswählt, liegt in ihrem Ermessen. ${ }^{17}$

Daneben kann die Kommission nach Art. 23 Abs. 2 VO 1/2003 ein Gesamtbußgeld verhängen, für welches mehrere Gesellschaften gesamtschuldnerisch haften. ${ }^{18}$ Dies ist mittlerweile die Regel. Die Gesamtschuld ist dabei zwar autonom unionsrechtlich zu verstehen, es handelt sich jedoch - im Gegensatz zu erstgenannten Vorgehensweise, für die strafrechtliche Garantien gelten müssen - um einen haftungsrechtlichen Ansatz. Auch hier kommt der Kommission ein Auswahlermessen zu. ${ }^{19}$ Es steht ihr also frei, ob sie die unmittelbar tatbeteiligte Gesellschaft oder (auch) andere Rechtsträger mit einer Geldbuße belegt. ${ }^{20}$ Nach Auffassung des EuGH hat die Kommission dabei nur die Haftungsanteile im Außenverhältnis zu bestimmen; die Festlegung, nach welchen Kriterien die Gesamtschuldner im Innenverhältnis haften, obliegt den nationalen Gerichten. ${ }^{21}$

Der europäische Unternehmensbegriff macht es auch möglich, Veränderungen des Unternehmens (Umstrukturierungen, Firmenumbenennung, Veräußerungen von Geschäftsbereichen etc.) als für den vom Unternehmen begangenen Rechtsverstoß unbeachtlich zu betrachten. Solange die wirtschaftliche Einheit, die die Zuwiderhandlung begangen hat, existiert, kann auf deren Rechtsträger als Bußgeldadressaten zugegriffen werden, mögen sie zwischenzeitlich auch umbenannt worden sein oder ihre Geschäftstätigkeit verändert haben.

Wenn eine Gesellschaft, die zum Zeitpunkt der Zuwiderhandlung Teil der wirtschaftlichen Einheit war, nach der Zuwiderhandlung veräußert wird, kann der Erwerber grundsätzlich zunächst nicht für die Zuwiderhandlung haftbar gemacht werden, selbst wenn er von dem Kartellverstoß Kenntnis hatte und nun mit der erworbenen Gesellschaft eine wirtschaftliche Einheit bildet. Voraussetzung für eine Haftung der Rechtsträger des erwerbenden Unternehmens ist, dass sie selbst einen

16 Siehe nur KOMM, 30.03.1983, AB1. L 317, S. 1 ff. (Gußeisen- und Gußstahlwalzen); DANNECKER Gerhard - FISCHER-FRITSCH, Jutta: Das EG-Kartellrecht in der Bussgeldpraxis. 1989, 264 ff. m.w.N.

17 Meist wurde in diesen Fällen lediglich die Muttergesellschaft bebußt, siehe DANNECKERFISCHER-FRITSCH (1989): 264 ff. m.w.N.

18 Siehe EuGH, 10. 04. 2014, Rs. C-231/11 P, www.curia.eu, Rn. 58 (Siemens AG Österreich).

19 BIEBER, Thomas: Die gesamtschuldnerische Haftung für die Zahlung von Kartellbussen im EU-Recht. 2014, 69 f. m.w.N.

20 SAUER, Ralf: In: SCHUlTE Josef L. - Just Christoph (Hrsg.): Kartellrecht. Art. 23 VO 1/2003, Rn. 8 m.w.N.; siehe nur EuG, Rs. T-71/03 u.a., Slg. 2005, II-10, Rn. 387 (Tokai Carbon); 17.05.2011, Rs. T-299/08, www.curia.eu, Rn. 60 ff. (Elf Aquitaine).

21 EuGH, 10. 04. 2014, Rs. C-231/11 P, www.curia.eu, Rn. 39 ff. (Siemens AG Österreich); 14. 04. 2014, Rs. C-247/11 P, Slg. 2011, II-633, Rn. 122 ff. (Areva SA); DE BRONETT, Georg: Gesamtschuldnerische Geldbußen nach EU-Kartellrecht für Personen, die wettbewerbswidrig handelnden ,wirtschaftlichen Einheiten“ angehören. EWS, 2015, 123, 128. 
Rechtsverstoß begeht, etwa, weil der erworbene Rechtsträger die Zuwiderhandlung fortsetzt. $^{22}$

Über die Kriterien der Kontinuität und Identität kann ausnahmsweise auch das einen Rechtsträger erwerbende Unternehmen, das selbst nicht am Kartellverstoß beteiligt war, dafür in Haftung genommen werden, wenn ansonsten in der wirtschaftlichen Einheit, die den Kartellverstoß begangen hat, kein Rechtsträger mehr vorhanden ist, gegen den die Geldbuße verhängt werden könnte und der erworbene Rechtsträger im Wesentlichen in dem neuen Unternehmen weiterexistiert. Vermieden werden soll damit, dass ein Rechtsträger in einem anderen Unternehmen aufgeht, um seiner Haftung zu entgehen und zugleich kein Bußgeldadressat existiert, der für die Geldbuße aufkommen könnte. ${ }^{23}$

Kritisiert wird an diesem Konzept der wirtschaftlichen Einheit vor allem die Weite des Unternehmensbegriffs und insbesondere die 100\%-Vermutung. ${ }^{24}$ Gegen Letztere wird eingewandt, dass sie der Unschuldsvermutung aus Art. 6 EMRK und Art. 48 Abs. 1 GRC zuwiderlaufe. ${ }^{25}$ Dieses Spannungsverhältnis erkennt zwar auch der Gerichtshof, er geht jedoch davon aus, dass die Vermutung sowohl dem effet utile als auch den rechtsstaatlichen Anforderungen genügt, weil die Vermutung zwar $\mathrm{uU}$ schwer, aber nicht unwiderleglich sei. ${ }^{26}$

Ebenfalls aus Effektivitätsgründen erweiterte der EuGH unlängst in einer bahnbrechenden Entscheidung den Anwendungsbereich des Unternehmensbegriffs auf Art. 11 der Richtlinie 2014/104/EU. ${ }^{27}$ In Sachen Sanska Industrial Solutions hatte der Gerichtshof - entgegen der ursprünglichen Intention des Richtliniengebers festgestellt, dass ,, der Begriff, Unternehmen 'im Sinne des Art. 101 AEUV, der einen autonomen Begriff des Unionsrechts darstellt, im Zusammenhang mit der Verhängung von Geldbußen durch die Kommission nach Art. 23 Abs. 2 der Verordnung Nr.

22 BIERMANN Jörg: In: IMMENGA, Ulrich - MESTMÄCKER, Ernst-Joachim (Hrsg.): Wettbewerbsrecht. 6. Aufl., 2019, Vor Art. 23 VO 1/2003 Rn. 114 f.

23 BIERMANn (2019): Vor Art. 23 VO 1/2003 Rn. 116 ff.; ZIMMER, Daniel: In: IMMENGA, Ulrich - MESTMÄCKER, Ernst-Joachim (Hrsg.): Wettbewerbsrecht. 6. Aufl., 2019, AEUV Art. 101 Abs. 1 Rn. 43.

24 Siehe nur THOMAS, Stefan: Die wirtschaftliche Einheit im EU-Kartellbußgeldrecht. $K S z W, 2011,10$ ff.; Voet VAn Vormizeele, Philipp: Die EG-kartellrechtliche Haftungszurechnung im Konzern im Widerstreit zu den nationalen Gesellschaftsrechtsordnungen. $W u W, 2010,1008,1009 \mathrm{ff}$.

25 Siehe nur BECHTOLD, Rainer - Bosch, Wolfgang: Der Zweck heiligt nicht alle Mittel. ZWeR, 2011, 160, $166 \mathrm{ff}$.

26 EuGH, 18. 07. 2013, Rs. C-501/11 P, www.curia.eu, Rn. 108 f. (Schindler); EuG, 17. 05. 2011, Rs. T-299/08, www.curia.eu, Rn. 60 (Elf Aquitaine); KeRsting, Christian: WuW, 2014, 1156, 1163.

27 Richtlinie 2014/104/EU des Europäischen Parlaments und des Rates v. 26. 11. 2014 über bestimmte Vorschriften für Schadensersatzklagen nach nationalem Recht wegen Zuwiderhandlungen gegen wettbewerbsrechtliche Bestimmungen der Mitgliedstaaten und der Europäischen Union. 
1/2003 keine andere Bedeutung als bei Schadensersatzansprüchen wegen des Verstoßes gegen die Wettbewerbsregeln der Union haben kann ". ${ }^{28}$

Darüber hinaus zeigt sich im europäischen Recht auch außerhalb des Kartellrechts die Tendenz zur Orientierung am kartellrechtlichen Unternehmensbegriff. ${ }^{29}$

\section{PARAllelentWicklung IM DeUTSCHEN KARTEllbUßGELDRECHT - VOM VERBANDSBUBGELDREGIME ZUR UNTERNEHMENSBEZOGENEN SANKTION DURCH DIE 9. GWB-NOVELLE}

\subsection{Vorbildcharakter des europäischen Unternehmenssanktionsregimes}

Trotz der teils noch offenen Fragen ist dieses europäische Sanktionsmodell infolge seiner Effektivität auch Vorbild für das Bußgeldregime, das mit der 9. GWB-Novelle in das deutsche Recht eingeführt wurde. Ziel des Gesetzgebers des Neunten Gesetzes zur Änderung des Gesetzes gegen Wettbewerbsbeschränkungen vom 1. 6. 2017, das am 9. 6. 2017 in Kraft getreten ist ${ }^{30}$, war es nämlich ausweislich der Gesetzesmaterialien, eine „unternehmensbezogene Sanktion“ einzuführen, um Kartellrechtsverstöße nach nationalem Recht ebenso effektiv und nachhaltig verfolgen zu können wie die EU-Kommission. ${ }^{31}$

\subsection{Unternehmensbezogene Sanktion auf eigenständigem nationalen Weg}

Durch die im Rahmen der Novellierung neu in das GWB aufgenommenen Regelungen der $\S 81 \mathrm{Abs}$. 3a bis 3e GWB ist es zu einer Erstreckung der bußgeldrechtlichen Verantwortung auf beherrschende Muttergesellschaften und Rechtsnachfolgegesellschaften und damit zu einer Ausweitung der bußgeldrechtlichen Verantwortung gekommen, mit der der Gesetzgeber zwar die Ergebnisse des europäischen Unternehmensbußgeldrechts erreichen wollte, allerdings auf einem eigenständigen nationalen Weg. Damit hat der nationale Gesetzgeber das gesellschaftsrechtliche Trennungsprinzip für das Kartellordnungswidrigkeitenrecht aufgeben und - vergleichbar dem europäischen Sanktionsregime - ein Unternehmenssanktionsrecht eingeführt, bei dem es für die Verhängung einer Geldbuße ausreicht, dass die juristische Person oder Personenvereinigung zum Zeitpunkt der Zuwiderhandlung der wirtschaftlichen Einheit angehört hat, der auch der Rechtsträger angehörte, dessen Leitungsperson die Zuwiderhandlung begangen hat. ${ }^{32}$ Mit $\S 81$

28 EuGH, 14. 03. 2019, Rs. C-724/17, www.curia.eu, Rn. 47 (Sanska Industrial Solutions).

29 V. BuSEKIST, Konstantin - FERDERMANN, Bernd - MÜLlER Nadja: Konzernverantwortung und Konzernhaftung. ZRFC, 2020, 263, 270 m.w.N.

30 BGBl. I 2017, 1416 ff.; JUNGBLUTH, Armin: Die 9. GWB-Novelle - Digitalisierung, SchlieBung der Wurstlücke, Kartellschadensersatz und anderes mehr... NZKart, 2017, 257.

31 DANNECKER Gerhard - MÜLLER Nadja: Das Kartellsanktionenrecht im Lichte der 9. GWB-Novelle. KSzW 2017, 125; MÄGER Thorsten - VON SCHREITTER Florian: Kartellordnungswidrigkeitenrecht nach der 9. GWB-Novelle. NZKart, 2017, 264, 265; JuNGBLUTH: NZKart, 2017, $257 \mathrm{f}$.

32 BT-Drucks. 18/10207, S. 88 f. 
Abs. 3a GWB sollte eine Regelung geschaffen werden, bei der sich schon auf der Verantwortungsebene die Frage stellt, welche Rechtsträger als Teil der wirtschaftlichen Einheit „Täter des Kartellverstoßes“" waren und deshalb zu ahnden sind. ${ }^{33}$

\subsection{Kritische Haltung der Literatur am eigenständigen deutschen Sanktions- regime}

Die herrschende Literatur geht dagegen von einer Fortgeltung des Trennungsprinzips aus und lehnt entsprechend die Einführung einer einheitlichen Unternehmensverantwortlichkeit ab: Auch wenn es nach dem Gesetz ausreiche, dass die juristische Person oder Personenvereinigung zum Zeitpunkt der Zuwiderhandlung der wirtschaftlichen Einheit angehört hat, der auch der Rechtsträger angehörte, dessen Leitungsperson die Zuwiderhandlung begangen hat, verbleibe es infolge der Rückbindung des $\S 81$ GWB an $\S 30$ OWiG bei der Verantwortung juristischer Personen und Verbände. ${ }^{34}$ Das Rechtsträgerprinzip bleibe also erhalten, wodurch es zu einer unglücklichen Vermengung von abgeleiteter und originärer Verantwortung komme, die verfassungsrechtlich bedenklich sei. ${ }^{35}$

In der Konsequenz hieraus folgt eine Unvereinbarkeit mit dem Schuldgrundsatz dahingehend, dass gegen die Muttergesellschaft, die einen bestimmenden Einfluss auf die kartellrechtswidrig handelnde Tochtergesellschaft ausgeübt hat, eine Geldbuße verhängt werden kann, obschon sich keine Leitungsperson der Muttergesellschaft selbst vorwerfbar verhalten hat. So aber werde eine am Kartellverstoß unbeteiligte juristische Personen sanktioniert ${ }^{36}$, was auf eine „Konzernzustandshaftung“ im Sinne einer kartellrechtlichen „Sippenhaft“ der Konzernobergesellschaf$\operatorname{ten}^{37}$ hinauslaufe, die verschuldensunabhängig eintrete. ${ }^{38}$

33 Vgl. Meyer-Lindemann, Hans Jürgen: In: KeRsting, Christian - PodsZun, Rupprecht (Hrsg.): Die 9. GWB-Novelle, 2017, 391 Rz. 56; s.a. DANNECKER, Gerhard: Das Kartellordnungswidrigkeitenrecht im Wandel: Von der Verbands- zur Unternehmensverantwortung? In: Festschrift für Rudolf Rengier zum 70. Geburtstag. 2018, 193, 198 f.

34 DANNECKER - MÜLleR: KSzW, 2017, 125, 127 f.; WeCK, Thomas: Sanktionen für Unternehmen: Theorie und Praxis. wistra, 2017, 169, 170.

35 DANNECKER (2018): a.a.O. 193, 199; TIMMERBEIL, Sven - BLOME, Max-Niklas: Steter Tropfen höhlt den Stein - Die „wirtschaftliche Einheit“ im deutschen Kartellrecht nach der 9. GWB-Novelle. $B B, 2017,1544,1547$.

36 MEYER-LiNDEMANN (2017): a.a.O. 394 Rz. 67.

37 BRETTEL, Hauke - THOMAS, Stefan: Der Vorschlag einer bußgeldrechtlichen „Konzernhaftung" nach $\S 81$ Abs. 3a RefE 9. GWB-Novelle. $W u W, 2016,336,337$.

38 DANNECKER - MÜLLER: $K S z W, 2017,125,128$; DANNECKER (2018): a.a.O., 193, 199 f.; HARNOS, Rafael: Harmonisierung des Kartellbußgeldrechts qua effet utile? ZWeR, 2016, 284, 304; MEYER-LiNDEMANN (2017): 394 Rz. 67. 


\subsection{Vermeidung von Friktionen nur bei Interpretation als Unternehmenssank- tionsregime unter Loslösung vom Trennungsprinzip}

Die Neukonzeptionierung des kartellrechtlichen Bußgeldregimes ist somit unterschiedlich interpretierbar und kann dem Vorwurf der Verfassungswidrigkeit nur dann entgehen, wenn man sie als eigenständige Ergänzung unter Beibehaltung der Verbandsverantwortlichkeit oder als Verbindung zweier Sanktionsmodelle zu einem einheitlichen, neuen Unternehmensverantwortungsmodell in Annäherung an das Unionsrecht betrachtet. Nur auf diese Weise lassen sich verfassungsrechtliche Friktionen vermeiden, da so das Unternehmen Sanktionsadressat ist, auf den der Schuldgrundsatz in Form des europäischen Grundsatzes der ,persönlichen“ Verantwortlichkeit Anwendung findet. ${ }^{39}$ Da nur das einheitliche Unternehmensverantwortlichkeitsmodell dem Schuldgrundsatz Rechnung trägt, ist eine entsprechende Interpretation im Wege der normerhaltenden verfassungskonformen Auslegung geboten. ${ }^{40}$ Maßgebend ist demnach die Verantwortung des Unternehmens als wirtschaftliche Tätigkeiten ausübende Einheit, ,, unabhängig von ihrer Rechtsform und der Art ihrer Finanzierung " ${ }^{41} .^{42}$ Die Ordnungswidrigkeit der Leitungsperson löst entsprechend die Verantwortlichkeit des Unternehmens aus, die es wiederum rechtfertigt, hierfür die Rechtsträger in Anspruch zu nehmen, wie es auch im europäischen Kartellordnungswidrigkeitenrecht der Fall ist. ${ }^{43}$

\subsection{Bewusstes Abweichen des deutschen Bußgeldregimes vom europäischen Modell}

An anderer Stelle weicht das deutsche Bußgeldregime jedoch bewusst vom EURecht ab und vermeidet damit die Probleme, die von der im europäischen Recht umstrittenen Vermutungsregel ausgehen. Laut Gesetzesbegründung zur 9. GWB-Novelle sind für den Nachweis des bestimmenden Einflusses von Mutter- und Tochtergesellschaft die Maßstäbe des deutschen Rechts maßgeblich; es bedürfe somit beweismäßig der vollen tatrichterlichen Überzeugung, die unter Heranziehung von Erfahrungssätzen gebildet werden könne, wobei in Fällen, in denen eine deutliche Mehrheit der Anteile gehalten wird, eine hohe Wahrscheinlichkeit dafür spreche, dass die Geschäftspolitik der betreffenden Gesellschaft tatsächlich durch die Mehrheitsanteilseignerin bestimmt wird. ${ }^{44}$ Die Abkehr von dieser Vermutungsregel ist in

39 Eingehend DANNECKER - MÜLlER: $K S z W, 2017,125,128$ ff.; DANNECKER (2018): a.a.O., 193, 200.

40 Dazu DANNECKER, Gerhard: In: Leipziger Kommentar. 12. Aufl., 2007, § 1 Rn. 374 ff.

41 So EuGH, 23.04.1991, Rs. C-41/90, Slg. 1991, I-1979 Rz. 21 (Höfner und Elser).

42 Siehe nur EuGH, 11. 12. 1997, Rs. C-55/96, Slg. 1997, I-7119 Rz. 21 (Job Centre); EuG, 13. 12. 2006, Rs. T-217/03 und T 245/03, Slg. 2006, II-4987 Rz. 52 (FNCBV); s.a. OST, Konrad - KALLFASS, Gunnar - ROESEN, Katrin: Einführung einer Unternehmensverantwortlichkeit im deutschen Kartellsanktionenrecht - Anmerkungen zum Entwurf der 9. GWB-Novelle. NZKart, 2016, 447, 457.

43 DANNECKER (2018): a.a.O., 193, $198 \mathrm{ff}$.

44 BT-Drucks. 18/10207, 90. 
Bezug auf die hier im europäischen Recht zu Recht geltend gemachten Bedenken durchaus zu begrüßen, wobei in der Literatur teils ein Gleichlauf in der Praxis mit dem europäischen Recht befürchtet wird. ${ }^{45}$ Darüber hinaus soll nach dem Willen des deutschen Gesetzgebers auch zwischen Schwestergesellschaften eine wirtschaftliche Einheit nicht angenommen werden. ${ }^{46}$

\subsection{Berechtigte Kritik am deutschen „Sonderweg“}

Tatsächlich kritisch zu betrachten an der Neukonzeptionierung im Rahmen der 9. GWB-Novelle ist zunächst die Einführung einer sog. Ausfallhaftung für die Übergangszeit in $\S 81 \mathrm{a}$ GWB, die verhindern soll, dass ein Bußgeld nicht festgesetzt oder vollstreckt werden kann, weil nach der Bekanntgabe der Einleitung des Bußgeldverfahrens der betroffene Rechtsträger erlischt oder Vermögen verschoben wird. ${ }^{47}$ Rechtsgrund für die Anordnung einer Ausfallhaftung ist nach der Vorstellung des Gesetzgebers ,,nicht die Kartellordnungswidrigkeit, sondern vielmehr ein der Tat nachgelagertes Ereignis, namentlich das nach Einleitung des Bußgeldverfahrens veranlasste Erlöschen einer nach \& $30 \mathrm{OWiG}$ verantwortlichen Gesellschaft oder eine anderweitige Verschiebung von Vermögen, die zur Folge hat, dass das Bußgeld nicht festgesetzt oder vollstreckt werden kann, also das Vermögen des Bußgeldverantwortlichen sich verringert. Ein ordnungswidrigkeitenrechtlicher Vorwurf gegen das Unternehmen ist damit, anders als bei der Neuregelung nach § 81 Abs. 3 a bis Abs. 3c, nicht verbunden. "48 Zutreffend wird an dieser Regelung kritisiert, dass obschon es sich nach dem Willen des Gesetzgebers hierbei um eine zivilrechtliche Haftung handeln soll ${ }^{49}$ - ebenfalls an das Konzept der wirtschaftlichen Einheit angeknüpft wird, so dass sich die Frage stellt, ob es sich bei der „Ausfallhaftung“ tatsächlich um ein haftungsrechtliches Instrument oder nicht tatsächlich doch um eine Sanktion handelt, auf die das strafrechtliche Rückwirkungsverbot Anwendung findet. ${ }^{50}$ Letzteres dürfte zu bejahen sein. ${ }^{51}$

45 Eingehend DANNECKER - MÜLLER: $K S z W, 2017,125,130$; DANNECKER (2018): a.a.O., 193, 201; TIMMERBEIL - BLOME: $B B, 2017,1544,1546$; siehe auch MEYER-LINDEMANN (2017): 392 f. Rz. 61 f., der die ausdrückliche Ablehnung der Vermutungswirkung im Gesetzesentwurf als ,hohle Pflichtübung “ sieht; kritisch auch HAUS, Florian C.: Neue Bußgeldhaftung für Konzerne: Die Ahndung von Kartellverstößen nach der 9. GWB-Novelle. Der Konzern, 2017, 381, 384 f.

46 BT-Drucks. 18/10207, 89.

47 NZKart-Beilage, 2017, 2, 35.

48 BT-Drucks. 18/10207, 95.

49 BT-Drucks. 18/10207, 95.

50 Siehe auch MEYER-LINDEMANN (2017): 406 f. Rz. $103 \mathrm{f}$.

51 Eingehend dazu DANNECKER - MÜLLER: KSzW, 2017, 125, 134 ff; HAUS: Der Konzern, 2017, 381, 388; von einer „Um-Etikettierung“ durch den Gesetzgeber sprechen auch MÄGER - V. SCHREITTER: NZKart, 2017, 264, 277; vgl. auch dies.: RefE zur 9. GWB-Novelle: Verfassungsrechtliche Bedenken gegen die Ausweitung einer bußgeldrechtlichen Konzernhaftung. DB, 2016, 2159, 2164. 
Ebenfalls kritisch zu sehen ist die mit der 9. GWB-Novelle eingeführte Erweiterung der Auskunfts- und Mitwirkungspflichten in $\S 81 \mathrm{~b}$ GWB (vormals $\S 81 \mathrm{a}$ a.F. GWB) mit Blick auf den Grundsatz der Selbstbelastungsfreiheit. Nach der Gesetzesbegründung soll die Erweiterung der Auskunftspflicht in $\S 81 \mathrm{~b}$ Abs. 1 Nr. 3 GWB eine effektive Aufklärung der Unternehmensstruktur und der dahinterstehenden Unternehmensträger gewährleisten. Da nunmehr neben den nach § $30 \mathrm{OWiG}$ verantwortlichen Rechtsträgern auch diejenigen eruiert werden müssten, die bestimmenden Einfluss im Unternehmen ausgeübt haben, müssten diese über die Auskunftspflicht identifizierbar gemacht werden. ${ }^{52}$ Insofern geht die Erzwingung von Auskünften über die des vormaligen $\S 81$ a GWB a.F. hinaus, da diese lediglich Informationen zur Umsatz- und damit zur Sanktionshöhe verlangten, welche man als ,außerhalb des engeren Kerns des sachlichen Schutzbereichs des nemo tenetur-Grundsatzes " betrachten konnte. ${ }^{53}$ Die Auskunftspflicht der Vorgängerregelung betraf daher nicht das "Tatwissen“ des beschuldigten Unternehmens, so dass ein Verstoß gegen die Selbstbelastungsfreiheit wohl noch nicht zwingend anzunehmen war, obschon bereits § 81a GWB a.F. wegen seiner staatlich erzwungenen Auskunftspflicht bezüglich u.U. sensiblen Unternehmensinformationen kritisch betrachtet wurde. ${ }^{54}$ Da die Erweiterung der Auskunftspflicht im Rahmen der Neuregelung aber explizit auch die Informationserlangung über die wirtschaftliche Einheit - also den Täter des Kartellverstoßes - bezweckt, ist ihre Vereinbarkeit mit dem nemo tenetur-Grundsatz fraglich. ${ }^{55}$ Die anstehende 10. GWB-Novelle geht nun noch einen Schritt weiter und sieht noch weitergehende Ausnahmen vom Auskunftsverweigerungsrecht i.S.d. § 55 StPO für natürliche Personen vor. So soll eine Auskunft dann nicht mehr verweigert werden können, wenn die Auskunft nur die Gefahr der Verfolgung im Kartellverfahren begründet und die Kartellbehörde der natürlichen Person im Rahmen ihres Ermessens eine Nichtverfolgungszusage erteilt hat. An dieser Nichtverfolgungszusage kritisch zu sehen ist jedoch, dass sie mangels gesetzlicher Regelung weder bindend sein dürfte noch die natürliche Person vor einer Verfolgung wegen anderer Ordnungswidrigkeiten oder Straftaten schützt. ${ }^{56}$

\subsection{Harmonisierung nach der ECN+-Richtlinie}

Unbenommen der teils am europäischen Unternehmensbegriff geübten Kritik macht die ECN+-Richtlinie aus dem Jahr 2018 nunmehr überdeutlich, dass es auch auf nationaler Ebene Geldbußen für Unternehmen und Unternehmensvereinigungen i.S.d.

52 BT-Drucks. 18/10207, 98.

53 Eingehend dazu DANNECKER, Christoph: Der nemo tenetur-Grundsatz - prozessuale Fundierung und Geltung für juristische Personen. ZStW 127 (2015), 307 ff.

54 DANNECKER, Gerhard - DANNECKER, Christoph - MÜLLER, Nadja: Das Kartellordnungswidrigkeitenrecht nach der 8. GWB-Novelle: weiterer Reformbedarf? ZWeR, 2013, 417, 437.

55 Eingehend DANNECKER - MÜLLER: $K S z W, 2017,125,137$ f; DANNECKER (2018): a.a.O., 193, 206.

56 Näher dazu KLUMPP, Ulrich: Änderungen im Bußgeldrecht und -verfahren nach der 10. GWB-Novelle. NZKart, 2020, 9, 12 f. 
europäischen Kartellrechts geben muss. So heißt es in Art. 13 Abs. 5 „,Die Mitgliedstaaten stellen sicher, dass für die Zwecke der Verhängung von Geldbußen gegen Muttergesellschaften sowie rechtliche und wirtschaftliche Nachfolger von Unternehmen der Begriff des Unternehmens angewandt wird ". ${ }^{57}$ Vor diesem Hintergrund scheint die Neukonzeptionierung des kartellrechtlichen Bußgeldregimes im Rahmen der 9. GWB-Novelle im Ansatz richtig.

\section{ERGEBNIS}

Denn trotz einigen berechtigten Kritikpunkten am Konzept der Kommission und der Europäischen Gerichte ist die Entwicklung und die damit verbundene Ausdehnung des funktionalen Unternehmensbegriffes insgesamt zu begrüßen: Sie wird der wirtschaftlichen Realität und der gesellschaftspolitischen Rolle von Unternehmen als „global player" gerecht. ${ }^{58}$

Insofern ist es als Schritt in die richtige Richtung zu werten, dass sich der deutsche Gesetzgeber vor dem Hintergrund des europäischen Harmonisierungsdrucks dazu entschlossen hat, eine Unternehmenssanktion im Rahmen der 9. GWB-Novelle einzuführen. ${ }^{59}$ Abzuwarten bleibt letztlich, ob das Konzept auf lange Sicht weitreichend genug ist, oder ob nicht die Verabschiedung vom Rechtsträgerprinzip - jedenfalls für das deutsche Kartellrecht - die konsequentere Alternative wäre. ${ }^{60}$

\section{LITERATURVERZEICHNIS}

[1] BIEBER, Thomas: Die gesamtschuldnerische Haftung für die Zahlung von Kartellbussen im EU-Recht. 2014.

[2] BeChTOLD, Rainer - Bosch, Wolfgang: Der Zweck heiligt nicht alle Mittel. ZWeR, 2011, 160-168.

[3] BIERMANN, Jörg: In: IMMENGA, Ulrich - MESTMÄCKER, Ernst-Joachim (Hrsg.), Wettbewerbsrecht. 6. Aufl., 2019.

[4] BRETTEL, Hauke - THOMAS, Stefan: Der Vorschlag einer bußgeldrechtlichen „Konzernhaftung“ nach $\S 81$ Abs. 3a RefE 9. GWB-Novelle. $W u W, 2016$, 336-340.

57 Richtlinie (EU) 2019/1 des Europäischen Parlaments und des Rates vom 11. Dezember 2018 zur Stärkung der Wettbewerbsbehörden der Mitgliedstaaten im Hinblick auf eine wirksamere Durchsetzung der Wettbewerbsvorschriften und zur Gewährleistung des reibungslosen Funktionierens des Binnenmarkts, ABlEU v. 14. 01. 2019, L11/3.

58 ORTMANN, Günther: Für ein Unternehmensstrafrecht: Sechs Thesen, sieben Fragen, eine Nachbemerkung. NZWiSt, 2017, 241, 249.

59 S.a. DANNECKER - MÜLLER: $K S z W, 2017,125,130$; a.A. BRETTEL - THOAMS: $W u W$, 2016, 336, 338, die die Bebußung der Muttergesellschaft wegen des Fehlens eines spezifischen Verhaltensbezuges als präventionspolitisch verfehlt ansehen.

60 Siehe dazu auch V. BUSEKIST - FEDERMANN - MÜLLER: ZRFC, 2020, 263 ff. 
[5] DANNECKER, Christoph: Der nemo tenetur-Grundsatz - prozessuale Fundierung und Geltung für juristische Personen. ZStW, 127 (2015), 370-409.

[6] DANNECKER, Gerhard: Das Kartellordnungswidrigkeitenrecht im Wandel: Von der Verbands- zur Unternehmensverantwortung? In: Festschrift für Rudolf Rengier zum 70. Geburtstag. 2018, 193-208.

[7] DANNECKER, Gerhard: In: Leipziger Kommentar. 12. Aufl., 2007.

[8] DANNECKER, Christoph - DANNECKER, Gerhard: Europäische und verfassungsrechtliche Vorgaben für das materielle und formelle Unternehmensstrafrecht. NZWiSt, 2016, 162-177.

[9] DANNECKER, Gerhard - FISCHER-FRITSCH, Jutta: Das EG-Kartellrecht in der Bussgeldpraxis. 1989.

[10] DANNECKER, Gerhard - DANNECKER, Christoph - MÜLLER, Nadja: Das Kartellordnungswidrigkeitenrecht nach der 8. GWB-Novelle: weiterer Reformbedarf? ZWeR, 2013, 417-447.

[11] DANNECKER, Gerhard - MÜLLER, Nadja: Kartellstraf- und -ordnungswidrigkeitenrecht. In: WABNITZ, Heinz-Bernd - JANOVSKY, Thomas - SCHMITT, Lothar (Hrsg.): Handbuch Wirtschafts- und Steuerstrafrecht. 5. Aufl., 2020, Kap. 19.

[12] DANNECKER, Gerhard - MÜLLER, Nadja: Das Kartellsanktionenrecht im Lichte der 9. GWB-Novelle. KSzW, 2017, 125-140.

[13] DE BRONETT, Georg: Gesamtschuldnerische Geldbußen nach EU-Kartellrecht für Personen, die wettbewerbswidrig handelnden ,wirtschaftlichen Einheiten" angehören. EWS, 2015, 123.

[14] HARNOS, Rafael: Harmonisierung des Kartellbußgeldrechts qua effet utile? ZWeR, 2016, 284-307.

[15] Haus, Florian C.: Neue Bußgeldhaftung für Konzerne: Die Ahndung von Kartellverstößen nach der 9. GWB-Novelle. Der Konzern, 2017, 381-388.

[16] JungBluth, Armin: Die 9. GWB-Novelle - Digitalisierung, Schließung der Wurstlücke, Kartellschadensersatz und anderes mehr... NZKart, 2017, 257-258.

[17] Kersting, Christian: Wettbewerbsrechtliche Haftung im Konzern. Der Konzern, 2011, 445-510.

[18] KeRsting, Christian: Die Rechtsprechung des EuGH zur Bußgeldhaftung in der wirtschaftlichen Einheit. $W u W, 2014,1156-1173$.

[19] KLuMPP, Ulrich: Änderungen im Bußgeldrecht und -verfahren nach der 10. GWB-Novelle. NZKart, 2020, 9-14. 
[20] MÄGER, Thorsten - VON SCHREITTER, Florian: RefE zur 9. GWB-Novelle: Verfassungsrechtliche Bedenken gegen die Ausweitung einer bußgeldrechtlichen Konzernhaftung. DB, 2016, 2159-2164.

[21] MÄGER, Thorsten - VON SCHREITTER, Florian: Kartellordnungswidrigkeitenrecht nach der 9. GWB-Novelle. NZKart, 2017, 264-278.

[22] MeYer-LindemanN, Hans Jürgen: Das neue Bußgeldrecht. In: Kersting, Christian - PoDSZUN, Rupprecht (Hrsg.): Die 9. GWB-Novelle. 2017, 371412.

[23] ORTMANN Günther: Für ein Unternehmensstrafrecht: Sechs Thesen, sieben Fragen, eine Nachbemerkung. NZWiSt, 2017, 241-251.

[24] OST, Konrad - KALLFASS Gunnar - ROESEN Katrin: Einführung einer Unternehmensverantwortlichkeit im deutschen Kartellsanktionenrecht - Anmerkungen zum Entwurf der 9. GWB-Novelle. NZKart, 2016, 447-458.

[25] SAUER, Ralf: In: SCHUlte, Josef, L. - Just, Christoph (Hrsg.): Kartellrecht. 2. Aufl., 2016.

[26] ThOMAs, Stefan: Die wirtschaftliche Einheit im EU-Kartellbußgeldrecht. $K S z W, 2011,10-14$.

[27] Weck, Thomas: Sanktionen für Unternehmen: Theorie und Praxis. wistra, 2017, 169-173.

[28] TimmerbeIL, Sven - Blome, Max-Niklas: Steter Tropfen höhlt den Stein Die "wirtschaftliche Einheit" im deutschen Kartellrecht nach der 9. GWBNovelle. BB, 2017, 1544-1550.

[29] Voet VAN VormizeELe, Philipp: Die EG-kartellrechtliche Haftungszurechnung im Konzern im Widerstreit zu den nationalen Gesellschaftsrechtsordnungen. $W u W, 2010,1008-1019$.

[30] V. BuseKIST, Konstantin - FedermanN, Bernd - MÜLLER, Nadja: Konzernverantwortung und Konzernhaftung. ZRFC, 2020, 263-270.

[31] ZIMMER, Daniel: In: IMMENGA, Ulrich - MESTMÄCKER, Ernst-Joachim (Hrsg.): Wettbewerbsrecht. 6. Aufl., 2019. 